\title{
ArcheoSciences
}

Revue d'archéométrie

$36 \mid 2012$

Varia

\section{Analyse in situ des dessins préhistoriques de la grotte de Rouffignac par fluorescence $\mathrm{X}$ et diffraction X portable}

In situ Analysis of Rock art in the Cave of Rouffignac by Using a Portable XRF-

XRD System

Lucile Beck, Hélène Rousselière, Jacques Castaing, Adrian Duran, Matthieu Lebon, Sophia Lahlil et Frédéric Plassard

\section{OpenEdition}

Journals

Édition électronique

URL : https://journals.openedition.org/archeosciences/3874

DOI : $10.4000 /$ archeosciences.3874

ISBN : 978-2-7535-2243-5

ISSN : 2104-3728

Éditeur

Presses universitaires de Rennes

Édition imprimée

Date de publication : 31 décembre 2012

Pagination : 139-152

ISBN : 978-2-7535-2241-1

ISSN : 1960-1360

Référence électronique

Lucile Beck, Hélène Rousselière, Jacques Castaing, Adrian Duran, Matthieu Lebon, Sophia Lahlil et Frédéric Plassard, « Analyse in situ des dessins préhistoriques de la grotte de Rouffignac par fluorescence X et diffraction X portable », ArcheoSciences [En ligne], 36 | 2012, mis en ligne le 31 décembre 2014, consulté le 01 février 2022. URL : http://journals.openedition.org/archeosciences/ 3874 ; DOI : https://doi.org/10.4000/archeosciences.3874 


\title{
Analyse in situ des dessins préhistoriques de la grotte de Rouffignac par fluorescence $X$ et diffraction $\mathrm{X}$ portable
}

\author{
In situ Analysis of Rock Art in the Cave of Rouffignac by Using \\ a Portable XRF-XRD System
}

\author{
Lucile Beck*,**, Hélène Rousselière*, Jacques Castaing*, Adrian Duran*, \\ Matthieu Lebon ${ }^{* * * *}$, Sophia LahliL ${ }^{*}$ et Frédéric Plassard ${ }^{* * * *}$
}

\begin{abstract}
Résumé : La Grotte de Rouffignac (Dordogne, France) est un site d’art paléolithique qui renferme de nombreux dessins réalisés au trait noir (mammouths, bisons, rhinocéros laineux, chevaux, bouquetins...). Bien qu’aucune datation directe n’ait été réalisée à ce jour, les œuvres graphiques de cette caverne sont en général rattachées au Magdalénien.

Un appareil portable couplant fluorescence et diffraction X, développé au Centre de recherche et de restauration des musées de France pour intervenir dans les musées ou sur des œuvres monumentales a été utilisé pour la première fois dans une grotte ornée. Les résultats obtenus en fluorescence X confirment les éléments manganèse, fer et baryum comme constituants principaux des pigments. Ils montrent aussi la présence d'autres éléments tels que le silicium, le titane, le chrome, le zinc et le potassium. La diffraction a permis d'identifier la présence de pyrolusite (dioxyde de manganèse), romanéchite et éventuellement de la hollandite, ces deux derniers composés étant des oxydes mixtes de baryum et de manganèse. La diffraction a aussi mis en évidence la présence systématique de quartz dans le pigment.

Cette campagne a démontré la possibilité d'effectuer des mesures de diffraction des rayons X en environnement difficile (accès aux parois, température, humidité) et a permis de réaliser les premiers diffractogrammes in situ directement sur des représentations préhistoriques. Les premiers résultats montrent que la Frise des mammouths de la galerie Breuil forme un ensemble de composition chimique relativement homogène, en cohérence avec la composition stylistique. En revanche, les figures du Grand Plafond sont de composition chimique différente plus variée.
\end{abstract}

\begin{abstract}
The Cave of Rouffignac (Dordogne, France) is a Palaeolithic rock art site which contains numerous black drawings (mammoths, bisons, rhinoceros, horses, ibexes...). Although no direct dating was carried out until now, the artworks of this cave are generally connected with the Magdalenian Culture. A portable system coupling X-ray fuorescence and X-ray diffraction developed at the C2RMF in Paris to work in museums or on monuments was used for the first time in a decorated cave. Results obtained with X-ray fluorescence confirm the main constituents of pigments : manganese, iron and barium. They also show the presence of other elements such as silicon, titanium, chromium, zinc and potassium. X-ray diffraction allowed identifying the presence of pyrolusite (manganese dioxide), romanechite and, eventually, hollandite. These two last compounds are barium manganese oxides. Quartz is also systematically observed in the pigment.

This campaign has demonstrated the possibility of performing X-ray diffraction in difficult environment (access to walls, temperature, and humidity) and has allowed to achieve the first diffractograms in situ, directly on prehistoric representations. The first results show that the Frieze of the mammoths of the gallery Breuil has a homogeneous chemical composition, in coherence with the stylistic composition. On the other hand, the figures of the Grand Plafond have more variable chemical composition.
\end{abstract}

* C2RMF - UMR171 CNRS, Centre de Recherche et de Restauration des Musées de France, Palais du Louvre, Porte des Lions, 14 quai FrançoisMitterrand, 75001 Paris, France.

** CEA, DEN, Service de Recherches de Métallurgie Physique, Laboratoire JANNUS, 91191 Gif-sur-Yvette, France. (lucile.beck@cea.fr)

*** Muséum National d'Histoire Naturelle, Département de Préhistoire, UMR 7194, 1 rue René-Panhard, 75013 Paris, France.

**** SARL Grotte de Rouffignac, 24580 Rouffignac-Saint-Cernin. (frederic.plassard@wanadoo.fr), UMR 5199 PACEA-Préhistoire, Paléoenvironnement, Patrimoine Université Bordeaux 1. 
Mots clé : Fluorescence X (XRF), diffraction X (XRD), Grotte de Rouffignac, art préhistorique, pigments préhistoriques, oxyde de manganèse, in situ, système portable.

Keywords : X-ray fluorescence X-ray diffraction Rouffignac Cave, prehistoric rock art, prehistoric pigment, manganese oxide, in situ, portable system.

Les auteurs tiennent à remercier Jean Plassard et Frédéric Goursolle de la Grotte de Rouffignac et Marie-Françoise Castaing pour leur précieuse aide pendant les expériences. Les campagnes ont été effectuées dans le cadre de l'ANR MADAPCA (Micro-Analyses et Datations de l'Art Préhistorique dans son Contexte Archéologique) dirigée par P. Paillet et avec les concours d'E. Man-Estier, M. Menu, I. Reiche et C. Vignaud.

L. Beck remercie J. de Sanoit et D. Chambellan du CEA de Saclay pour avoir mis leurs données à sa disposition.

\section{INTRODUCTION}

\section{La grotte de Rouffignac}

La grotte de Rouffignac est l'un des sites majeurs de l'art paléolithique. Reconnue en 1956, elle a fait l'objet de nombreuses études visant dans un premier temps à certifier son authenticité (Breuil, 1959; Nougier et Robert, 1957) et dans un deuxième temps à étudier les dessins et gravures, non seulement d'un point de vue archéologique mais aussi pour en comprendre l'élaboration et veiller à leur conservation (Barrière, 1982; Plassard, 1999; Plassard, 2005). Quoique parfaitement inscrite dans l'art pariétal paléolithique, la caverne présente quelques spécificités techniques, thématiques et stylistiques. Techniquement, les 240 figurations animales se répartissent entre la gravure (fine au silex, ou plus profonde, au bâtonnet ou au doigt) et le dessin. Celui-ci est systématiquement noir et concerne une centaine d'images essentiellement regroupées sur le Grand Plafond et dans la galerie Henri Breuil. Cinq espèces animales sont bien représentées : cheval (16), bison (29), bouquetin (12), rhinocéros (11) et mammouth (159). C'est l'omniprésence de ce dernier qu'on doit retenir, puisqu'il est habituellement assez secondaire dans les grottes ornées et qu'il constitue ici plus de $60 \%$ des figurations. La fréquence et la clarté des panneaux construits en frise sont un dernier élément remarquable de ce dispositif pariétal. Si le Grand Plafond réunit 65 images sans qu'aucune organisation d'ensemble puisse apparaitre, la Frise des dix mammouths, celle des Trois rhi- nocéros ou celle du Salon Rouge forme autant de panneaux parfaitement structurés.

\section{Caractérisation chimique des pigments}

Une première étude chimique des pigments constituants les dessins de la grotte de Rouffignac a été effectuée de manière non destructive en 2004. Grâce à la mise au point d'un appareil de fluorescence des rayons X (XRF) portable, de Sanoit et al. (2005) ont pu réaliser les premières analyses de fluorescence $\mathrm{X}$ de dans une grotte. Ils ont ainsi déterminé la composition élémentaire de dix figures qui se sont avérées être essentiellement constituées de manganèse, baryum et fer. Les auteurs concluaient eux-mêmes sur la nécessité de compléter leur étude par l'identification des phases minéralogiques. En effet, si les diverses phases d'oxyde de manganèse (tableau 1) peuvent être distinguées par leur composition chimique, notamment par la présence d'éléments tels que le baryum ou le potassium, elles le sont surtout par leur structure et l'état de valence de l'ion $\mathrm{Mn}$ (Chalmin, 2003, Jezequel et al., 2011). Par exemple, une grande variété d'oxydes de manganèse - cryptomélane, todorokite, pyrolusite, romanéchite et hollandite - utilisés seuls ou sous forme de mélange a été observée pour la grotte de Lascaux (Chalmin et al., 2003). D'autres sites ont aussi été étudiés, comme Pech-Merle où de la hollandite et éventuellement de la romanéchite ont été identifiées dans les prélèvements de la Frise Noire. L'existence d'un oxyde hydraté $\mathrm{Al}_{5} \mathrm{Mn}_{13} \mathrm{O}_{28}, 8 \mathrm{H}_{2} \mathrm{O}$ a été également relevée par Guineau et al., 2001.

Pour avoir accès à ces phases minérales in situ, il faut avoir recours à des appareils portables de spectrométrie Raman (Ospitali et al., 2006, Lahlil et al., accepté) ou de diffraction X (XRD). Dans le cadre du projet européen Eu-Artech un appareil a été développé au C2RMF combinant XRF et diffraction des rayons X (Gianoncelli et al., 2008). Cet ensemble, mis au point pour caractériser les matériaux employés pour les œuvres d'art exposées dans les musées, a été employé pour la première fois en grotte ornée.

Deux missions ont été menées dans la grotte de Rouffignac avec pour objectif principal l'étude des pigments des des- 


\begin{tabular}{|c|c|c|}
\hline $\begin{array}{l}\text { Mineralogical } \\
\text { name }\end{array}$ & Formula & Symmetry \\
\hline Manganite & $\mathrm{MnOOH}$ & Monoclinic \\
\hline Pyrolusite & $\mathrm{MnO}_{2}$ & Tetragonal \\
\hline Bixbyite & $\mathrm{Mn}_{2} \mathrm{O}_{3}$ (or natural $(\mathrm{Mn}, \mathrm{Fe})_{2} \mathrm{O}_{3}$ ) & Cubic \\
\hline Hausmannite & $\mathrm{Mn}_{3} \mathrm{O}_{4}$ & Tetragonal \\
\hline Romanechite & $\mathrm{Ba}_{2} \mathrm{Mn}_{5} \mathrm{O}_{10} \cdot x \mathrm{H}_{2} \mathrm{O}$ & Monoclinic \\
\hline Hollandite & $\mathrm{BaMn}_{8} \mathrm{O}_{16} \cdot x \mathrm{H}_{2} \mathrm{O}$ & Monoclinic \\
\hline Cryptomelane & $(\mathrm{K}, \mathrm{Ba}) \mathrm{Mn}_{8} \mathrm{O}_{16} \cdot x \mathrm{H}_{2} \mathrm{O}$ & Monoclinic \\
\hline Todorokite & $(\mathrm{Ba}, \mathrm{Ca}, \mathrm{K}, \mathrm{Na}) \mathrm{Mn}_{6} \mathrm{O}_{12} \cdot x \mathrm{H}_{2} \mathrm{O}$ & Monoclinic \\
\hline
\end{tabular}

Tableau 1 : Principaux oxydes de manganèse naturels (Chalmin, 2003).

Table 1: Main natural manganese oxides.

sins préhistoriques présents sur les parois de la cavité. La première mission visait à tester l'appareillage en condition souterraine et à effectuer les premiers tests sur pigment et paroi ; la deuxième mission a permis de compléter le corpus des dessins analysés.

\section{CONDITIONS EXPÉRIMENTALES}

\section{Configuration des lieux et accès}

La grotte de Rouffignac est une grotte spacieuse aménagée pour recevoir du public (fig. 1). Elle est alimentée en électricité et le petit train permet d'acheminer le matériel dans les zones d'intérêt. Cependant l'humidité et la fraîcheur des lieux $\left(95 \%\right.$, température $\left.13{ }^{\circ} \mathrm{C}\right)$ pouvaient nous faire craindre des problèmes sur l'alimentation Haute Tension du tube, ou comme lors de la mission de de Sanoit et al. (2005) la condensation d'eau sur le détecteur XRF. Nous n'avons pas rencontré de telles difficultés et les appareillages ont fonctionné correctement. Pour pouvoir atteindre les parois décorées situées soit au plafond, soit en creux à environ 1.5-2 m de hauteur, il a été nécessaire d'installer l'appareil sur une grue mobile. Grâce à la fixation solide des appareils de mesure et la conception ingénieuse du montage qui rend solidaire tous les composants, il a été possible de manipuler la grue et d'approcher l'ensemble XRF-XRD à quelques

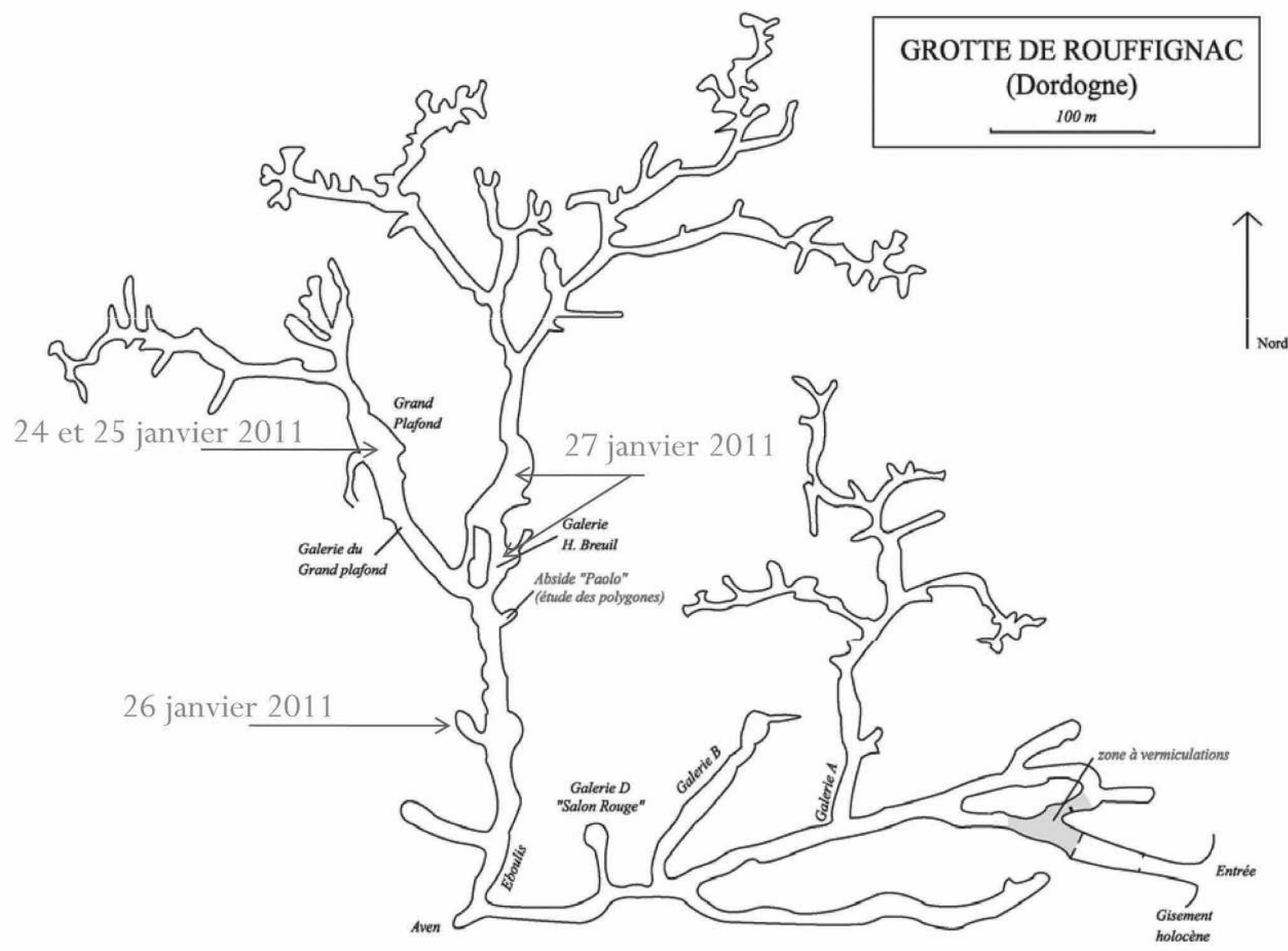

Figure 1 : Plan de la Grotte de Rouffignac.

Figure 1: Map of the Rouffignac cave. 
millimètres des figures sans dommage ni pour les appareils ni pour la paroi. Les mesures s'effectuent sans contact, à une distance d'environ $15 \mathrm{~mm}$ entre le détecteur XRF et la paroi (fig. 2).

Deux sites ont été sélectionnés pour les analyses : la galerie Henri Breuil et le Grand plafond. Dans la galerie Breuil, la Frise des dix mammouths forme un panneau homogène dans lequel deux groupes de mammouths sont disposés en vis-à-vis. Les silhouettes tracées en lignes noires mesurent environ $1 \mathrm{~m}$ et se répartissent à hauteur d'homme sur un bandeau de paroi compris entre un entablement rocheux à la base et un banc de nodules de silex au sommet. Si la partie supérieure des images est parfaitement lisible, le développement d'efflorescences de calcite a fortement altéré la moitié inférieure des œuvres. Proche de la Frise des dix mammouths, un panneau représente 3 rhinocéros.
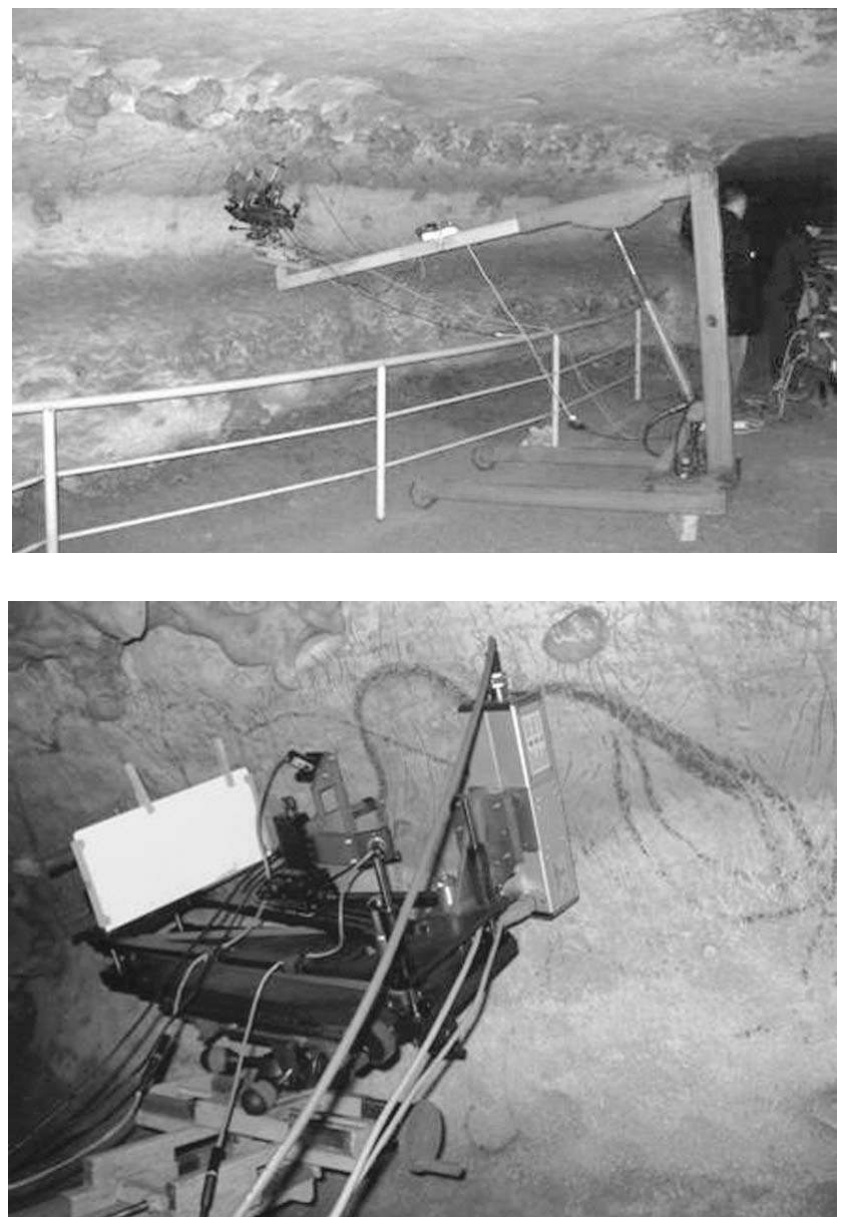

Figure 2 : L'appareil de XRF-XRD en cours de mesure (a) vue d'ensemble (b) vue rapprochée.

Figure 2: XRF-XRD system during the experiment (a) overview (b) detail.
Le Grand plafond, second locus étudié, est composé, à l'inverse de la galerie Breuil, d'une grande diversité de dessins. C'est un ensemble plus riche et beaucoup plus varié. Soixante-cinq représentations y sont accumulées sur une quarantaine de $\mathrm{m}^{2}$. C'est le seul endroit de la caverne offrant une telle densité d'images et une telle diversité puisque mammouths, bisons, chevaux, bouquetins et rhinocéros y sont associés et parfois entremêlés.

Au total, 15 figures ont été étudiées, les 10 mammouths de la galerie Henri Breuil formant un groupe a priori homogène qu'il est pertinent de comparer aux rhinocéros de la même galerie, et aux dessins du Grand Plafond (4 analysés) (tableau 2). Compte tenu de la difficulté d'accès de certaines représentations, il n'a pas été possible d'atteindre des figures qui offraient cependant un intérêt certain.

\section{Le système portable XRF/XRD}

L'appareil portable XRF-XRD est particulièrement adapté à l'identification et à la caractérisation de matériaux in-situ (Gianoncelli et al. 2008). Souvent en effet, les œuvres d'art ne peuvent être déplacées et les échantillonnages ne sont pas toujours possibles. De plus, les techniques XRF et XRD sont totalement non destructives.

L'appareil (fig. 3) comprend :

- une source de rayons $\mathrm{X}(30 \mathrm{~W}, 40 \mathrm{keV}$ et $700 \mu \mathrm{A})$ de IFG (iMOX micro-foyer) constituée d'un tube avec une anode en cuivre (raie $\mathrm{K}-\alpha$ du cuivre à $\mathrm{E}=8.05 \mathrm{keV}$ ) refroidi à l'air, isolé à l'aide une fenêtre en béryllium de $0,01 \mathrm{~mm}$ et équipé de 2 filtres (nickel ou aluminium),

- des optiques polycapillaires qui permettent d'obtenir un faisceau parallèle d'environ $3 \mathrm{~mm}$ de diamètre,

- deux pointeurs laser rouge de faible puissance (0,9 MW) pour les alignements,

différentes fentes verticales (de largeurs : $0.075-0.1-$ $0.2-0,5 \mathrm{~mm}$ ),

- un dispositif supportant la plaque-image (Imaging Plate) enregistrant les diffractogrammes,

- un détecteur SDD (Silicon Drift Detector) de $7 \mathrm{~mm}^{2}$ collectant les rayons $\mathrm{X}$ de fluorescence et situé perpendiculairement à la paroi.

En plus du système portable XRF/XRD, un scanner (DenOptix GENDEX) est utilisé pour numériser les « imaging plates». Les acquisitions de fluorescence $\mathrm{X}$ et le traitement des diffractogrammes sont effectués à partir d'un ordinateur et à l'aide de différents logiciels :

- ADMCA et PymCa (Solé et al., 2007) pour la fluorescence $\mathrm{X}$,

- Fit2D pour obtenir les diagrammes de diffraction et DIFFRACT ${ }^{\text {plus }}$ EVA pour interpréter ces diffractogrammes. 


\begin{tabular}{|c|c|c|c|}
\hline \multicolumn{4}{|c|}{ Galerie Henri Breuil } \\
\hline $\begin{array}{c}\text { Référence } \\
\text { (Barrière, 1982) }\end{array}$ & Description & $\mathrm{Nb}$ de points réalisés & Campagne \\
\hline MAM 190 & $1^{\text {er }}$ mammouth de la frise & 1 & Janvier 2011 \\
\hline MAM 191 & $2^{\mathrm{e}}$ mammouth & 1 & Janvier 2011 \\
\hline MAM 192 & $3^{\mathrm{e}}$ mammouth & 2 & Janvier 2011 \\
\hline MAM 193 & $4^{\mathrm{e}}$ mammouth & 3 & Janvier 2011 \\
\hline MAM 194 & $5^{\mathrm{e}}$ mammouth & 2 & $\begin{array}{c}\text { Janvier } 2011 \\
\text { Novembre } 2009\end{array}$ \\
\hline MAM 195 & $6^{\mathrm{e}}$ mammouth & 2 & Janvier 2011 \\
\hline MAM 196 & $7^{\mathrm{e}}$ mammouth & 1 & Janvier 2011 \\
\hline MAM 197 & $8^{\mathrm{e}}$ mammouth & 2 & Janvier 2011 \\
\hline MAM 198 & $9^{\mathrm{e}}$ mammouth & 1 & Janvier 2011 \\
\hline MAM 199 & $\begin{array}{l}10^{\mathrm{e}} \text { et dernier mammouth } \\
\text { de la frise }\end{array}$ & 1 & Novembre 2009 \\
\hline RH 185 & $3^{\mathrm{e}}$ rhinocéros & 1 & Novembre 2009 \\
\hline \multicolumn{4}{|c|}{ Grand Plafond } \\
\hline $\begin{array}{c}\text { Référence } \\
\text { (Barrière, 1982) }\end{array}$ & Description & Nb de points réalisés & Campagne \\
\hline BISON 100 & Bison & 3 & Janvier 2011 \\
\hline BOUQ 102 & Bouquetin & 3 & Janvier 2011 \\
\hline MAM 107 & Mammouth & 2 & Janvier 2011 \\
\hline MAM 66 & Mammouth & 4 & Novembre 2009 \\
\hline MAM 66 & Trait & 2 & Novembre 2009 \\
\hline
\end{tabular}

Tableau 2 : Dessins de la grotte de Rouffignac analysés par XRF-XRD portable.

Table 2 : Analysed drawings of the Rouffignac cave by portable XRF-XRD.

(6) Echantillon

\section{(2) Laser}

(4) Détecteur XRF

(3) Source $X$

(1) Laser

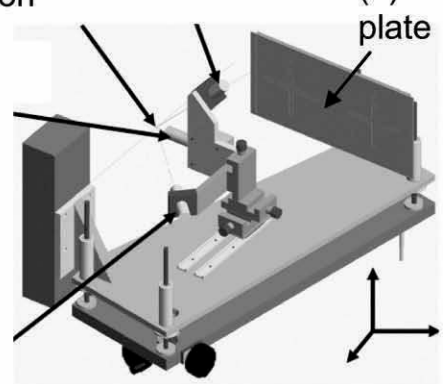

(5) Imaging plate

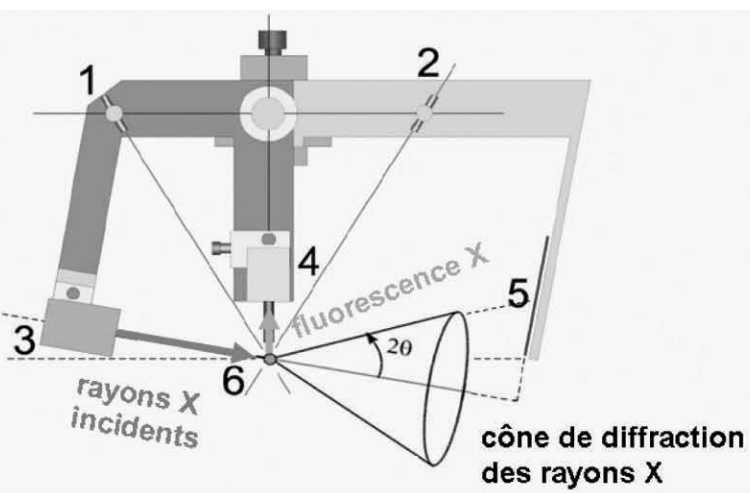

Figure 3 : (Voir planche couleur) Système portable XRF/XRD développé au C2RMF (a) Schéma (b) Vue de haut -1 et 2 : lasers de positionnement, 3 : tube de RX, 4 : Détecteur XRF, 5 : Imaging Plate, 6 : Point d'analyse Figure 3: (Voir planche couleur) C2RMF XRF-XRD portable system (a) Schematic view (b) Top view- 1 et 2 : lasers for positioning, $3: X$-ray tube, 4:XRF detector, 5: Imaging Plate, 6: Analysis spot 
L'angle d'incidence du faisceau de rayons $\mathrm{X}$ sur la paroi est d'environ $10^{\circ}$ ce qui permet une analyse rasante de la couche picturale. Cette configuration est particulièrement bien adaptée au cas des dessins sur paroi rocheuse puisqu'elle minimise la pénétration des rayons $\mathrm{X}$ dans le substrat en calcite. Le cliché de diffraction obtenu provient d'une couche d'environ $25 \mu \mathrm{m}$ d'épaisseur sous la surface pour des éléments légers $(\mathrm{Al}, \mathrm{Si}, \mathrm{K})$ et de $10 \mu \mathrm{m}$ pour des éléments lourds $(\mathrm{Pb}, \mathrm{Hg}, \mathrm{Sn})$. La taille du faisceau des rayons $\mathrm{X}$ est d'environ $3 \mathrm{~mm}$ de diamètre. La diffraction est régie par la loi de Bragg : $2 \mathrm{~d} \sin \mathrm{q}=\mathrm{n} \mathrm{l}=\mathrm{n}(1.24 / \mathrm{E}), \mathrm{d}(\mathrm{nm})$ étant la distance interréticulaire, $\mathrm{q}=$ l'angle d'incidence avec les plans réticulaires, $1(\mathrm{~nm})$ la longueur d'onde des rayons $\mathrm{X}$ et E l'énergie en $\mathrm{keV}$. Les rayons diffractés sont émis dans un cône $2 q$ (fig. $3 b$ ) enregistré sur l'imaging plate (fig. 4a) d'où l'on extrait le diffractogramme (fig. 4b).

Pour les dessins de la grotte de Rouffignac, chaque point de mesure comporte les étapes suivantes :

- déplacement de la grue au plus près de la paroi ornée,

- réglage sur le point d'analyse matérialisé par deux lasers rouges, à l'aide des translations verticales, et horizontales de manière à respecter la géométrie fixée pour une analyse de fluorescence X quantitative et pour les conditions de diffraction,

- démarrage de l'acquisition par l'ouverture de l'obturateur du tube de rayons $\mathrm{X}$ et enregistrement du spectre de fluorescence $\mathrm{X}$ pendant 5 minutes en simultané avec l'im-

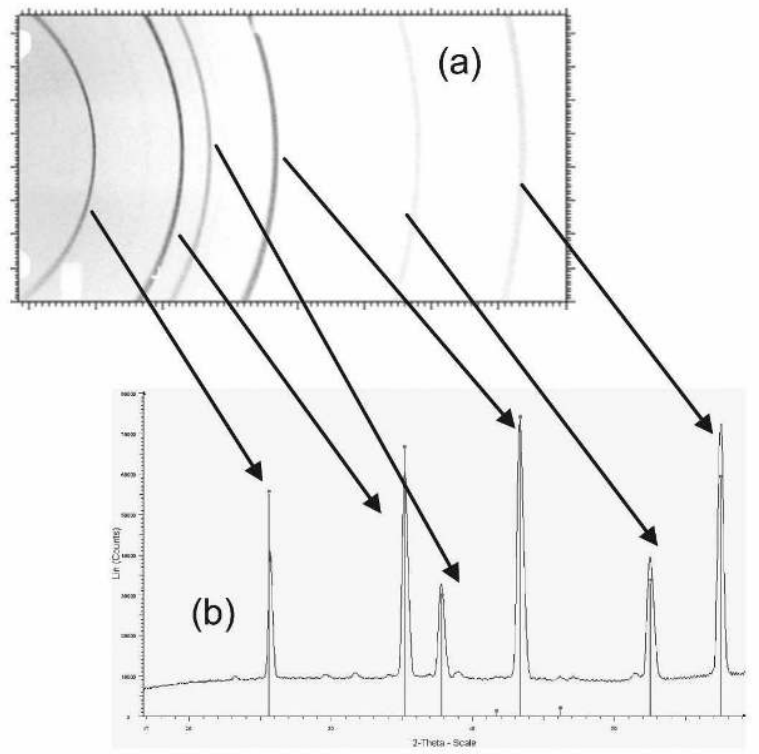

Figure 4 : (a) «Imaging Plate » et (b) diagramme obtenus sur une céramique en alumine.

Figure 4: (a) "Imaging Plate" and (b) X-ray diffractogram for an alumina sample. pression des anneaux de diffraction sur l'imaging plate pendant 20 à 30 minutes,

- numérisation de l'imaging plate et enregistrement du diffractogramme.

Ces opérations, effectuées pour chaque mesure sur dessin, ont été précédées par la mesure d'un "blanc " de paroi de manière à distinguer au mieux les éléments provenant du pigment de ceux de la calcite formant le substrat des dessins. A titre d'exemple, les signaux de la paroi et du dessin du Mammouth 194 sont superposés sur la figure 5. La comparaison fait ressortir les principaux éléments constitutifs du pigment : Si, K, Ba, Mn, Fe. Sur la figure 6a est présenté le diffractogramme obtenu sur le bison 100 montrant les anneaux attribuables au pigment constitué de pyrolusite et de quartz (fig. 6b). Les autres anneaux sont principalement dus à la calcite sous jacente.

\section{RÉsultats}

\section{Fluorescence des rayons $\mathrm{X}$}

Les spectres XRF enregistrent à la fois les rayons $\mathrm{X}$ émis par le pigment et ceux provenant de la paroi calcaire sousjacente. Afin de ne sélectionner que les éléments constitutifs du pigment nous avons effectué plusieurs points d'analyse sur le mammouth 66 (fig. 7). Ces points ont permis de mettre en évidence des corrélations positives entre le manganèse, élément majeur du pigment et les mineurs et traces suivants : K, Ti, Cr, Fe, Zn, Ba, et $\mathrm{Si}$ (fig. 8). On peut donc attribuer ces éléments au pigment; ils sont principalement liés à l'oxyde de manganèse comme élément du réseau cristallin $(\mathrm{Ba}, \mathrm{K})$ ou cation de substitution $(\mathrm{Fe}, \mathrm{Cr}$,

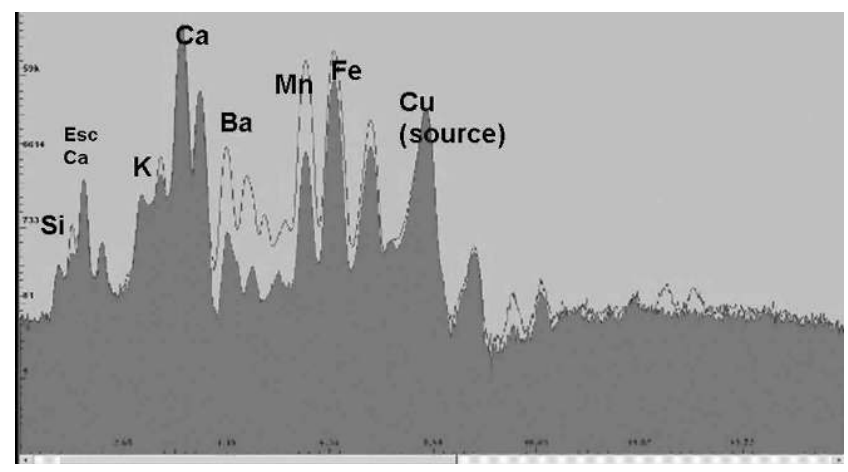

Figure 5 : Spectres XRF du mammouth 194 (trait pointillé) et de la paroi à proximité (gris).

Figure 5: XRF spectra obtained on the mammoth drawing \# 194 (dotted line) and on the rock nearby (grey). 


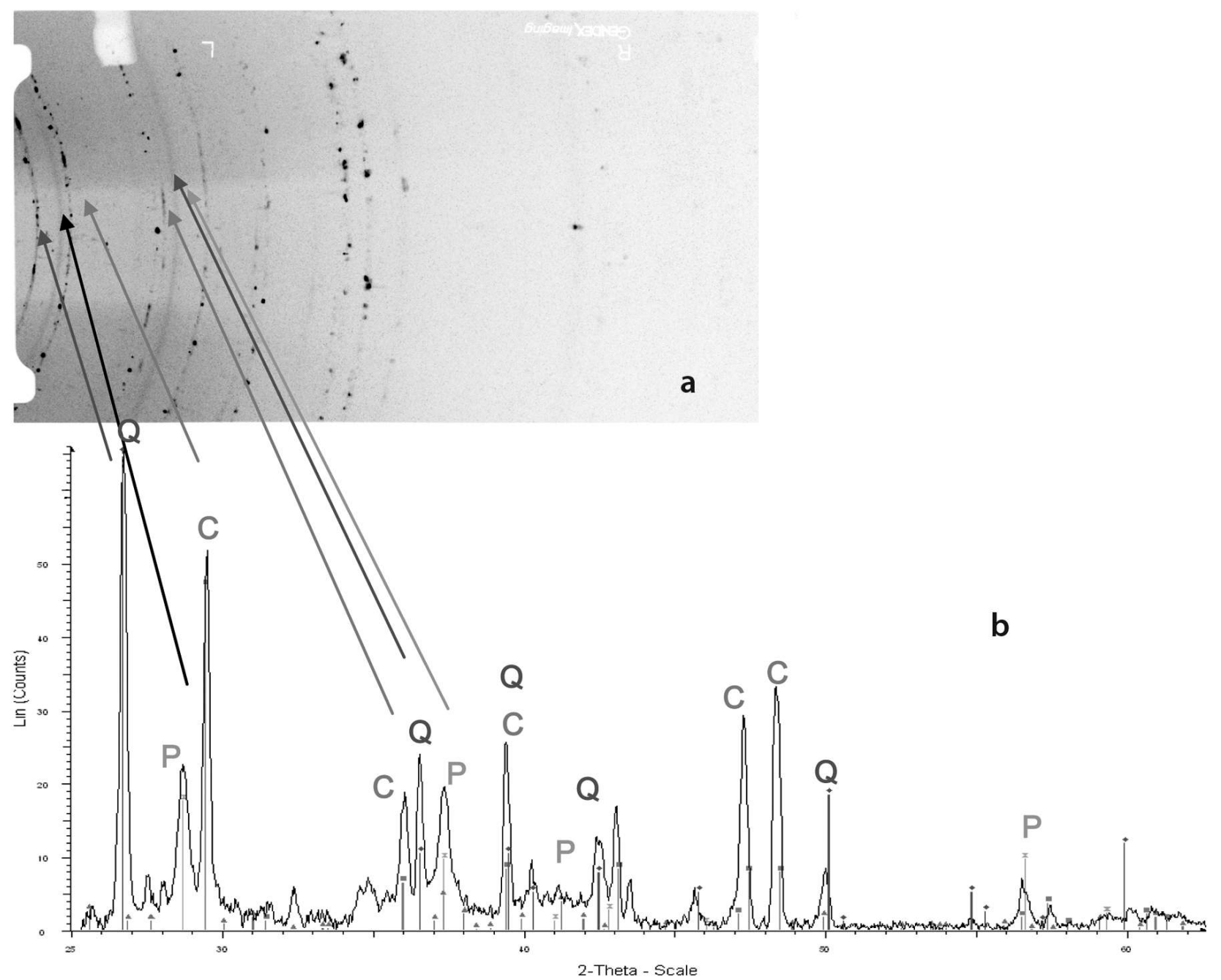

Figure 6: (Voir planche couleur) Clichés de XRD du bison 100 (a) «imaging plate » (b) diffractogramme. Phases observées : quartz (Q) et pyrolusite $(\mathrm{P})$ pour le pigment, calcite $(\mathrm{C})$ pour la paroi sous-jacente

Figure 6: (See colour plate) XRD obtained on the bison \# 100 (a) "imaging plate " (b) diffractogram. Observed phases : quartz (Q) and pyrolusite (P) for the pigment, calcite (C) for the back rock

Figure 7 : (Voir planche couleur) Points analysés sur le Mammouth 66 du Grand plafond

Figure 7: (See colour plate) Analysed spots on the mammoth drawing \# 66 of the Great Ceiling

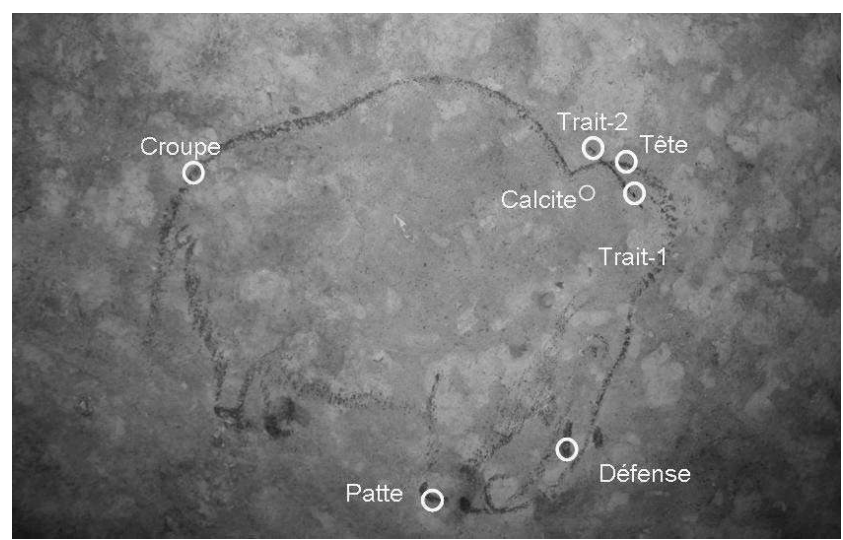

ArcheoSciences, revue d'archéométrie, 36, 2012, p. 139-151 


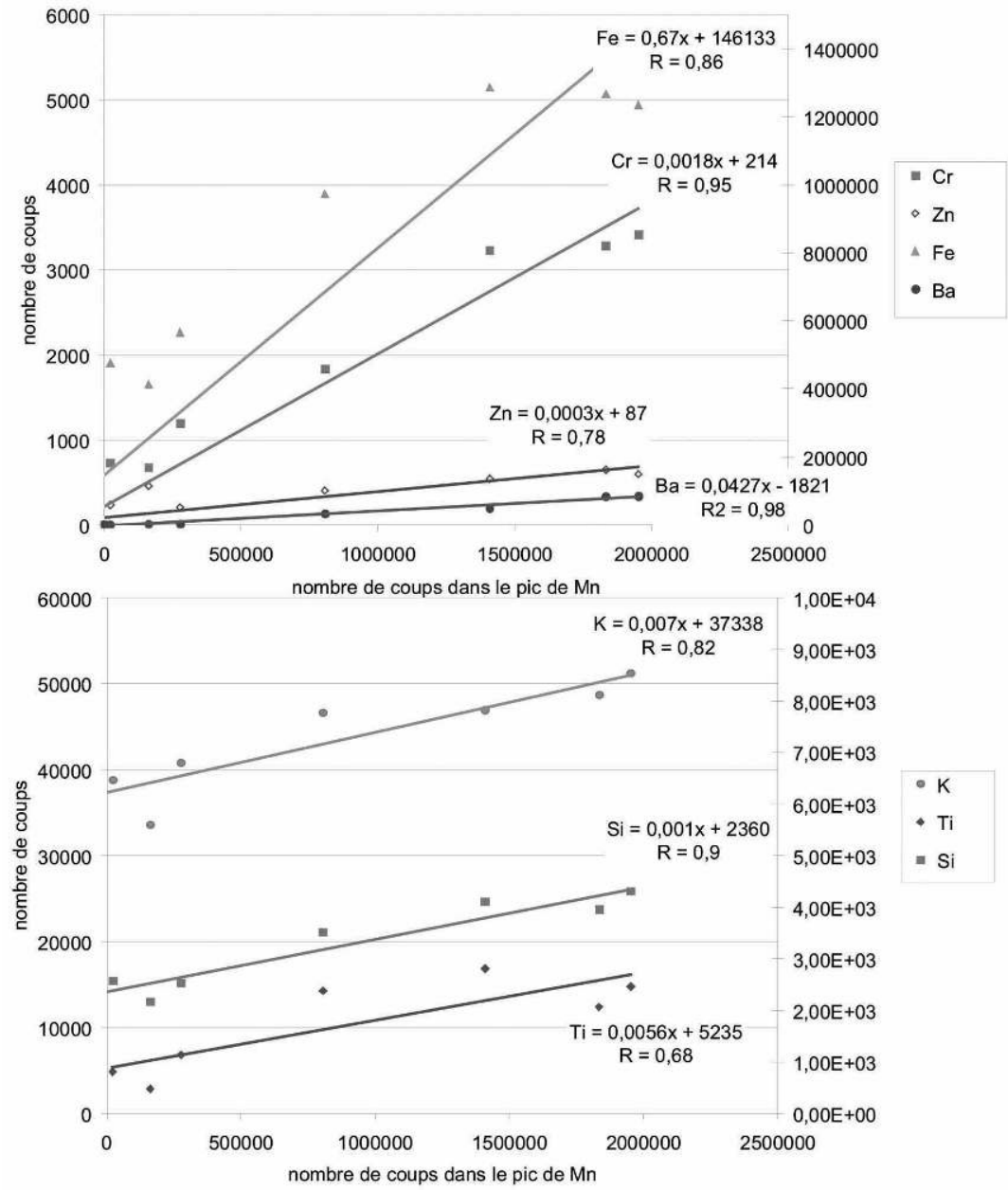

Figure 8: (Voir planche couleur) XRF sur 6 points du mammouth 66 et sur un point de la paroi : variation des différents éléments chimiques en fonction de la quantité de manganèse

Figure 8: (See colour plate) XRF results obtained on 6 different spots on mammoth drawing \# 66 and on the wall: element variations as a function of the manganese signal
Ti). Ils pourraient aussi, à l'exception du $\mathrm{Ba}$, avoir pour origine une phase argileuse contenue dans le pigment, mais en quantité assez faible ou trop mal cristallisée pour être observée en diffraction. En ce qui concerne l'élément Fe, on peut aussi envisager un apport de la paroi : de nombreux points effectués sur la calcite montrent que celle-ci peut aussi contenir du fer en teneur variable. Cependant, compte tenu de l'incidence rasante du dispositif, nous avons négligé cet apport. Le Si provient majoritairement de la présence de grains de quartz contenus dans le pigment, visibles à la loupe et observés seulement sur les diffractogrammes de pigment (cf. diffraction infra).

Pour comparaison avec l'étude de de Sanoit et al. (2005), nous avons retenu les éléments majeurs manganèse, fer et baryum. Les concentrations sont exprimées sous forme d'oxyde et bouclées à $100 \%$. Les résultats sont groupés dans le tableau 3. Sur les 15 figures analysées, la moyenne est de $52 \pm 13 \%$ de $\mathrm{MnO}_{2}, 38 \pm 13 \%$ de $\mathrm{Fe}_{2} \mathrm{O}_{3}$ et $9 \pm 3 \%$ de $\mathrm{BaO}$. Ces valeurs sont compatibles avec des oxydes de manganèse contenant des phases argileuses à l'état naturel.
Sur la figure 9 sont reportés les résultats des analyses en fonction de l'emplacement des dessins.

On remarque que la frise des mammouths forme un ensemble relativement homogène, distinct des dessins du Grand Plafond. Les teneurs en $\mathrm{BaO}$ (Tableau 4) confirment cette différence entre les 2 groupes avec des moyennes de $10 \pm 3 \%$ pour la Frise des mammouths et de $6 \pm 1 \%$ pour le Grand Plafond. Pour ce dernier, seul se détache le bouquetin 102 qui contient de forte teneur en $\mathrm{BaO}: 18 \pm 2 \%$. Quant aux deux rhinocéros de la galerie Breuil, leur signature chimique est plus proche des figures analysées du Grand Plafond que de la frise des mammouths. 


\begin{tabular}{|c|c|c|c|c|c|c|}
\hline Figure & \begin{tabular}{c|}
$\%$ \\
$\mathrm{MnO} 2$
\end{tabular} & $\begin{array}{c}\% \\
\text { Fe2O3 }\end{array}$ & $\begin{array}{c}\% \\
\mathrm{BaO}\end{array}$ & $\begin{array}{l}\text { Phase pigment } \\
\text { majeure }\end{array}$ & $\begin{array}{c}\text { Phase pigment } \\
\text { mineure }\end{array}$ & Phase paroi \\
\hline \multicolumn{7}{|c|}{ Galerie Henri Breuil } \\
\hline MAM190-01 & 59 & 29 & 12 & Quartz, Romanéchite, Pyrolusite & & Calcite \\
\hline MAM191-01 & 46 & 45 & 9 & Quartz, Romanéchite? Pyrolusite? & & Calcite \\
\hline MAM192-01 & 54 & 36 & 10 & Quartz, Romanéchite, Pyrolusite & & \\
\hline MAM192-02 & 46 & 46 & 8 & Quartz & & Calcite \\
\hline MAM193-02 & 53 & 32 & 15 & Quartz, Romanéchite? & & Calcite \\
\hline MAM193-03 & 47 & 41 & 12 & \multicolumn{3}{|c|}{ Diffractogramme non exploitable } \\
\hline MAM194-00 & 50 & 39 & 11 & Quartz & Non identifiée & Calcite \\
\hline MAM 194 (de Sanoit et al., 2005) & 58 & 28 & 14 & \multicolumn{3}{|c|}{ 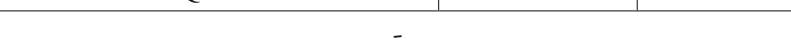 } \\
\hline MAM195-01 & 44 & 49 & 7 & \multicolumn{3}{|c|}{ Diffractogramme non exploitable } \\
\hline MAM195-02 & 48 & 43 & 9 & Quartz, Pyrolusite, Romanéchite? & Goethite? & Calcite \\
\hline MAM196-01 & 41 & 50 & 9 & Non identifiée & & Calcite \\
\hline MAM197-01 & 51 & 42 & 7 & Quartz, Pyrolusite, Romanéchite & Goethite & Calcite \\
\hline MAM197-03 & 49 & 43 & 8 & \multicolumn{3}{|c|}{ Diffractogramme non exploitable } \\
\hline MAM198-01 & 36 & 57 & 7 & Non identifiée & & Calcite \\
\hline MAM199-00 & 67 & 18 & 14 & \multicolumn{3}{|c|}{ Diffractogramme non exploitable } \\
\hline MAM199 (de Sanoit et al., 2005) - moyenne & 68 & 24 & 8 & \multicolumn{3}{|l|}{-} \\
\hline RH $185-00$ & 66 & 29 & 5 & Quartz, Pyrolusite & Romanéchite? & Calcite \\
\hline RH 185 (de Sanoit et al., 2005) - moyenne & 83 & 12 & 5 & & & \\
\hline \multicolumn{7}{|c|}{ Grand Plafond } \\
\hline MAM-66-croupe & 63 & 33 & 5 & \multicolumn{3}{|c|}{ Diffractogramme non exploitable } \\
\hline MAM-66-défense & 56 & 39 & 5 & \multicolumn{3}{|c|}{ Diffractogramme non exploitable } \\
\hline MAM-66-tête & 68 & 24 & 7 & Quartz, Hollandite ou Pyrolusite & & Calcite \\
\hline MAM-66-patte & 66 & 26 & 8 & Quartz, Hollandite ou Pyrolusite & & Calcite \\
\hline Bison100-01 & 67 & 27 & 6 & \multicolumn{3}{|c|}{ Diffractogramme non exploitable } \\
\hline Bison100-04 & 63 & 29 & 7 & Pyrolusite, Quartz & Romanéchite & Calcite \\
\hline Bison 100 - (de Sanoit et al., 2005) & 94 & - & 6 & \multicolumn{3}{|l|}{-} \\
\hline Bouq102-01 & 63 & 18 & 19 & Quartz & & \\
\hline Bouq102-02 & 62 & 20 & 18 & Quartz, Romanéchite & & Calcite \\
\hline Bouq102-03 & 61 & 20 & 19 & Quartz & & \\
\hline Bouq102- (de Sanoit et al., 2005) & 69 & 17 & 14 & \multicolumn{3}{|l|}{-} \\
\hline MAM107-01 & 66 & 28 & 6 & Quartz & & Calcite \\
\hline MAM107-02 & 61 & 34 & 5 & Quartz & & Calcite \\
\hline MAM107 - (de Sanoit et al., 2005) & 79 & 16 & 5 & \multicolumn{3}{|l|}{-} \\
\hline
\end{tabular}

Tableau 3 : Résultats de XRF et XRD sur les dessins de la grotte de Rouffignac.

Table 3: XRF-XRD results obtained on the drawings of the Rouffignac cave.

\begin{tabular}{|l|l|l|l|}
\hline $\begin{array}{l}\text { Incertitude relative (de Sanoit et } \\
\text { al., 2005) }\end{array}$ & $\pm 10 \%$ & $\pm 10 \%$ & $\pm 10 \%$ \\
\hline Incertitude relative (cette étude) & $\pm 10 \%$ & $\pm 20 \%$ & $\pm 20 \%$ \\
\hline
\end{tabular}




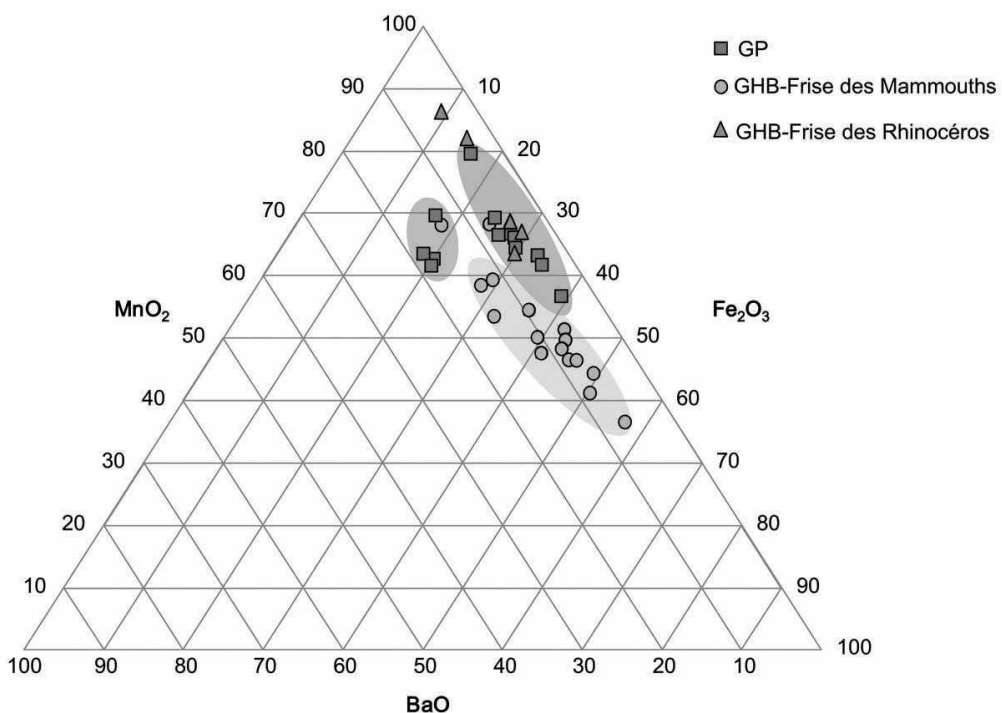

Figure 9: (Voir planche couleur) Compositions en $\mathrm{MnO}_{2}, \mathrm{Fe}_{2} \mathrm{O}_{3}$ et $\mathrm{BaO}$ des dessins de la grotte de Rouffignac mesurées par XRF. Le diagramme inclut les données de de Sanoit et al. (2005)

Figure 9: (See colour plate) $\mathrm{MnO}_{2}, \mathrm{Fe}_{2} \mathrm{O}_{3}$ and $\mathrm{BaO}$ contents for the drawings of the Rouffignac cave measured by XRF. Data of de Sanoit et al. (2005) are included

\begin{tabular}{|c|c|c|c|c|}
\hline & & $\% \mathrm{MnO} 2$ & $\% \mathrm{Fe} 2 \mathrm{O} 3$ & $\% \mathrm{BaO}$ \\
\hline Frise des mammouths - Galerie Breuil & Moyenne & 51 & 39 & 10 \\
\hline & Écart-type & 9 & 10 & 3 \\
\hline Grand Plafond sauf Bouq 102 & & & & \\
\hline & Moyenne & 65 & 28 & 6 \\
\hline Bouquetin 102 & Écart-type & 6 & 7 & 1 \\
\hline & Moyenne & 64 & 19 & 18 \\
\hline Frise des rhinocéros - Galerie Breuil & Écart-type & 4 & 2 & 2 \\
\hline & & & & \\
\hline & Écart-type & 9 & 10 & 3 \\
\hline
\end{tabular}

Tableau 4 : Synthèse des résultats de XRF sur les dessins de la grotte de Rouffignac.

Table 4: Summary of the XRF results obtained on the drawings of the Rouffignac cave.

\section{Diffraction des rayons $\mathrm{X}$}

En raison des difficultés d'alignement et de la faible cristallinité des pigments, il n'a pas été possible d'obtenir des clichés de diffraction exploitables pour toutes les figures. Sur les 30 diffractogrammes enregistrés, une douzaine a mis en évidence des phases minérales reliées au manganèse. Deux composés ont été identifiés avec certitude : la pyrolusite $-\mathrm{MnO}_{2}$ (fig. 6 et $11 \mathrm{~b}$ ) et la romanéchite $-\mathrm{Ba}_{2} \mathrm{Mn}_{5} \mathrm{O}_{10}$ (fig. 10). Un $3^{\text {e }}$ composé pourrait être la hollandite $\mathrm{BaMn}_{8} \mathrm{O}_{16}$ (fig. 11a). Dans ce dernier cas, la détermination est particulièrement délicate car pyrolusite et hollandite ont des angles de diffraction très proches, avec cependant des pics moins nombreux pour la pyrolusite, ce qui conduit à des signaux plus fins.
Dans le cas de la Frise des mammouths, on observe pour tous les dessins un mélange des deux oxydes pyrolusite et romanéchite. Selon la figure analysée, le composé majoritaire semble être la romanéchite (MAM 190, 191, 192, 193) ou la pyrolusite (MAM 195, 197).

En ce qui concerne les figures du Grand plafond, on retrouve les mêmes différences déjà relevées en fluorescence $\mathrm{X}$ : le bison 100 est composé de pyrolusite (fig. 6) alors que le bouquetin 102 est clairement constitué de romanéchite quasi pure (fig. 10). Le diffractogramme du mammouth 66 est plus difficile à interpréter : deux composés - pyrolusite et hollandite - correspondent aux angles détectés (fig. 11).

Le quartz est systématiquement présent dans tous les clichés de diffraction du pigment. Les diffractogrammes de blancs effectués sur paroi n'en contiennent pas, ce qui 
Figure 10 : (Voir planche couleur) Cliché de XRD du bouquetin 102. Phases observées : quartz (Q) et romanéchite $(\mathrm{R})$.

Figure 10: XRD (See colour plate) obtained on the ibex \# 102. Observed phases : quartz (Q) and romanechite $(R)$

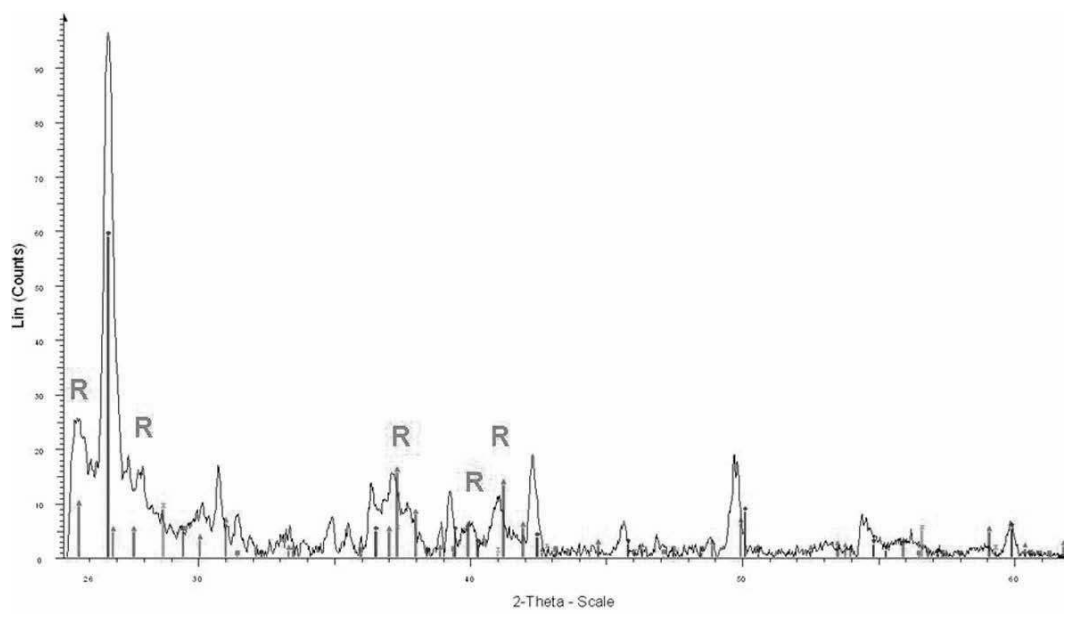

Figure 11 : (Voir planche couleur) Cliché de XRD du mammouth 66. Deux interprétations sont possibles pour le composé manganèse : (a) hollandite $(\mathrm{H})$ ou (b) pyrolusite $(\mathrm{P})$. Dans les deux cas, présence de quartz $(\mathrm{Q})$ provenant du pigment et de calcite (C) de la paroi.

Figure 11: (See colour plate) XRD obtained on the mammoth drawing \# 66. Two compounds are possible: (a) hollandite $(H)$ or (b) pyrolusite (P). In both cases, quartz (Q) from the pigment and calcite (C) from the rock are also observed.
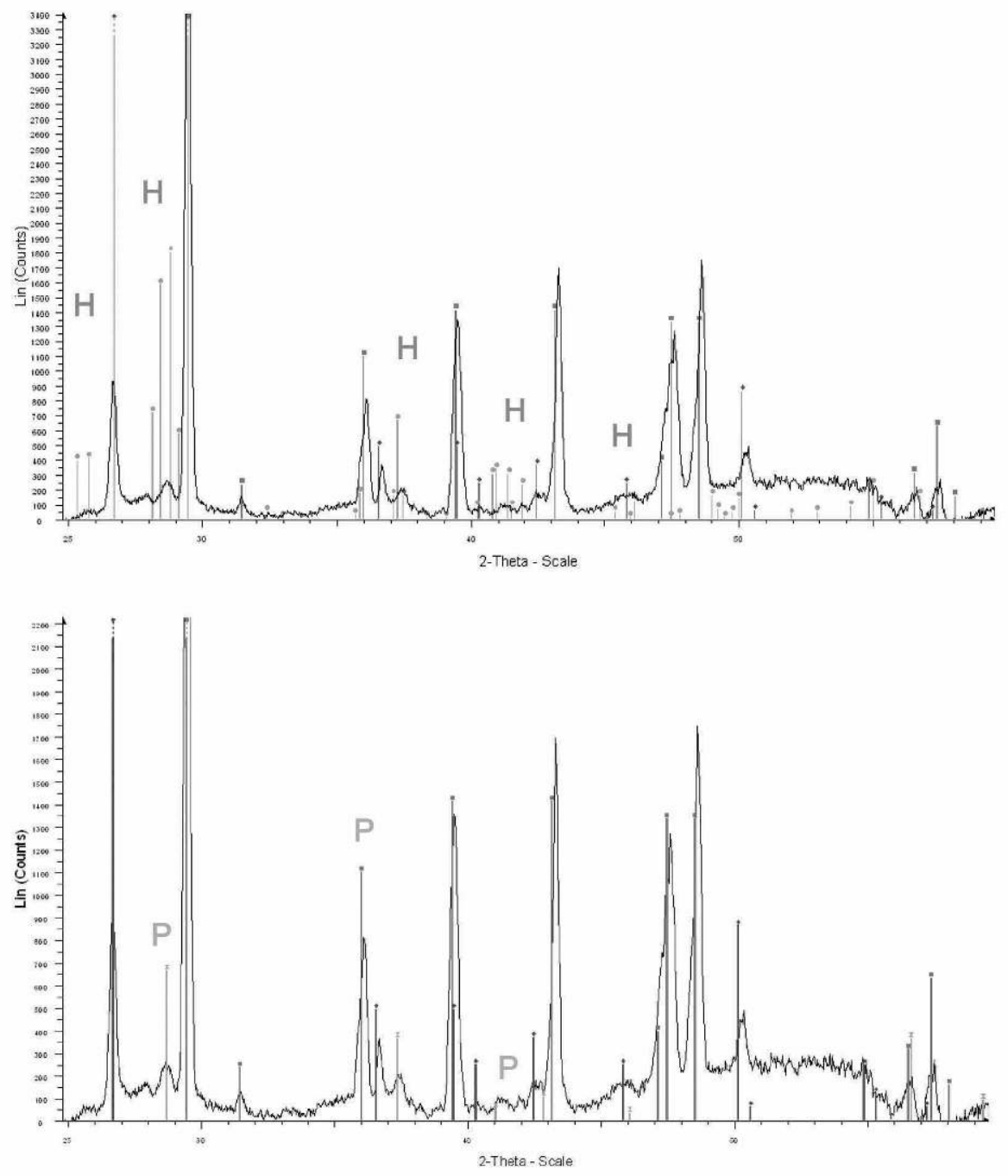
démontrent qu'il est bien un constituant du pigment. Un signal de la calcite est parfois présent sur les diffractions; celui-ci est dû à la paroi de la grotte.

\section{Synthèse}

D’une manière générale, on constate une bonne corrélation entre la phase minérale majoritaire et la teneur en baryum (tableau 5) : les dessins effectués à base de romanéchite $\left(\mathrm{Ba}_{2} \mathrm{Mn}_{5} \mathrm{O}_{10}\right)$ contiennent entre 15 à $19 \%$ de $\mathrm{BaO}$, les mélanges entre 7 et $10 \%$ et lorsque la pyrolusite $\left(\mathrm{MnO}_{2}\right)$ domine, on obtient 5-6\% de $\mathrm{BaO}$. Cette valeur non nulle de $\mathrm{BaO}$ est probablement due à des phases minoritaires peu cristallisées, contenant du baryum et présentes avec la pyrolusite.

L’ensemble des résultats montrent que :

La Frise des mammouths de la galerie Breuil forme un ensemble relativement homogène, de composition $50 \%$ $\mathrm{MnO}_{2}-40 \% \mathrm{Fe}_{2} \mathrm{O}_{3}-10 \% \mathrm{BaO}$ environ et constitué d'un mélange, très probablement naturel (car fréquemment observé), de pyrolusite et romanéchite $\left[\mathrm{MnO}_{2}+\right.$ $\left.\mathrm{Ba}_{2} \mathrm{Mn}_{5} \mathrm{O}_{10}\right]$.

Les oxydes de manganèse du Grand Plafond sont moins riches en oxyde de baryum (env. $6 \%$ pour Mam66, Mam107, Bison100 et Mam 121 (donnée de de Sanoit et al., 2005 pour cette dernière figure) à l'exception du bouquetin 102 (18\%). Ces différences sont corrélées à la présence d'oxydes distincts : un pigment à base de pyrolusite a été employé pour la plupart des figures analysées alors que le bouquetin 102 a été réalisé à partir d'une autre phase minérale : de la romanéchite quasiment "pure ».
La Frise des rhinocéros de la galerie Breuil (Rh185, cette étude; Rh183, de Sanoit et al., 2005) présente une signature chimique et minéralogique proche de celle des dessins du Grand plafond : faible baryum (5\%) et pyrolusite.

\section{ConClusion}

Grâce aux campagnes menées dans la grotte de Rouffignac, nous avons pu montrer la faisabilité et l'utilité du système XRD-XRF portable pour l'analyse in situ de pigments sur les parois d'une grotte ornée.

Malgré des conditions environnementales difficiles, l'ensemble des instruments liés à la XRF-XRD portable s'est comporté de manière satisfaisante permettant l'acquisition de données dans des conditions analogues à celles rencontrées dans les musées. Cependant la variabilité de la géométrie de l'environnement (sol incliné, parois arrondies, etc.) a ralenti le positionnement de l'appareil de mesures et a limité la quantité de mesures. L'accès aux parois n'aurait pas été possible sans le système de grue développé par les propriétaires de la grotte, qui a permis d'accéder au mieux aux différentes parois, d'inclinaison et de courbure variable. De plus, la grotte de Rouffignac est équipée en réseau électrique, ce qui n'est pas le cas de toutes les cavités.

En ce qui concerne les dessins préhistoriques de la grotte de Rouffignac, nous pouvons conclure que :

- La Frise des mammouths de la galerie Breuil forme un ensemble de composition chimique relativement homogène, en cohérence avec la " composition stylistique ». La Frise des dix mammouths est l'un des plus spectaculaires exemples de

\begin{tabular}{|c|c|c|}
\hline \multicolumn{4}{|c|}{ Figure } & \% BaO moyen & Oxydes de Mn \\
\hline MAM190 à 192 & 10 & Romanéchite + Pyrolusite \\
\hline MAM193 & 15 & Romanéchite? \\
\hline MAM195 & 9 & Pyrolusite + Romanéchite \\
\hline MAM197 & 7 & Pyrolusite + Romanéchite \\
\hline RH 185-00 & 5 & Pyrolusite \\
\hline \multicolumn{4}{|c|}{ Grand Plafond } & $\begin{array}{c}\text { Hollandite } \\
\text { ou Pyrolusite }\end{array}$ \\
\hline MAM-66 & 6 & Pyrolusite \\
\hline Bison100-04 & 7 & Romanéchite \\
\hline Bouq102-02 & 19 & \\
\hline Romanéchite & Référence & \\
\hline Pyrolusite & Env. 20\% & \\
\hline & Env. 0 \% & \\
\hline
\end{tabular}

Tableau 5 : Teneur en $\mathrm{BaO}$ mesurée par XRF et identification de l'oxyde de manganèse par XRD sur 10 dessins de la grotte de Rouffignac.

Table 5: BaO content measured by XRF and manganese oxide characterization by XRD for 10 drawings og the Rouffignac Cave. 
composition dans l'art pariétal paléolithique. L'unité thématique, technique et stylistique comme le traitement de tous les sujets à la même échelle et leur répartition régulière et symétrique sur la paroi laissent imaginer une unité de conception et de réalisation. La relative homogénéité chimique et cristallographique mise en évidence au sein de ce panneau par cette étude vient clairement à l'appui de cette hypothèse.

- Les figures du Grand Plafond sont en revanche de composition chimique plus variable avec d'une part, de faibles teneurs en $\mathrm{BaO}$ pour la plupart des figures (Mam66, Mam107, Bison100 et Mam 121 et d'autre part, des teneurs très élevées pour le Bouq102. Les figures du Grand Plafond (65 sujets sur $40 \mathrm{~m}^{2}$ ) n'offrent aucune organisation d'ensemble (diversité thématique, variété d'échelle de traitement, orientation variable des sujets, superpositions). Il n'est donc pas totalement surprenant qu'en dépit du nombre limité d'images étudiées, les résultats montrent une certaine dispersion. Cependant, le cas du bouquetin 102 est intéressant parce qu'il participe d'un petit ensemble de trois bouquetins. Une étude ultérieure pourra s'attacher à reconnaitre le degré d'homogénéité chimique des pigments de ces images. Plus largement, toutes les figurations de bouquetins de Rouffignac se trouvent sur le Grand Plafond et une étude stylistique détaillée de ces images est en préparation (Plassard, à paraître). Une confrontation des résultats avec des mesures complémentaires par XRF et XRD permettra peut-être de mieux comprendre les processus de mise en place des ouvres du Grand Plafond.

- La Frise des rhinocéros de la galerie Breuil est plus proche en composition chimique des dessins du Grand Plafond (Mam66, Mam107, Bison100 et Mam 121) que de la Frise des mammouths pourtant située dans la même galerie. À ce stade, les analyses sont trop peu nombreuses sur ces deux panneaux pour que ces résultats soient interprétables archéologiquement. Là encore, de nouvelles analyses sur le troisième rhinocéros de la frise ainsi que sur les rhinocéros du Grand Plafond sont à envisager.

\section{Bibliographie}

BArrière, Cl., 1982 - L'art pariétal de Rouffignac, Picard, 208 p., 519 fig.

Breuil, H., 1959 - Des preuves de l'authenticité des figures pariétales de la caverne de Rouffignac. Bulletin de la Société Préhistorique Française, t.56, p. 82-91.

Chalmin, E., 2003 - Caractérisation des oxydes de manganèse et usage des pigments noirs au Paléolithique supérieur. Thèse de doctorat, Université de Marne-La-Vallée, France.
Chalmin, E., Menu, M. et Vignaud, C., 2003 - Meas. Sci. Technol, 14, p. 1590-1597.

de Sanoit, J., Chambellan, D. et Plassard, F., 2005 Caractérisation in situ du pigment noir de quelques œuvres pariétales de la Grotte de Rouffignac à l'aide d'un système portable d'analyse par fluorescence X (XRF), ArchéoSciences 29, p. 61-68.

Gianoncelli, A., Castaing, J., Ortega, L., Doorhyée, E., Salomon, J., Walter, P., Hodeau, J.-L et Bordet, P., 2008 A portable instrument for in situ determination of the chemical and phase composition of cultural heritage objects, $X$-Ray Spectrometry 37, p. 418-423.

Guineau, B., Lorblanchet, M., Gratuze, B., Dulin, L., Roger, P., Akrish, R. et Muller, F., 2001 - Manganese black pigments in prehistoric paintings : the case of the black frieze of Pech Merle (France), Archaeometry 43, p. 211-225.

Jezequel, P., Wille, G., Beny, C., Delorme, F., Jean-Prost, V., Cottier, R., Breton, J., Dure, F. et Despriee, J., 2011 - Characterization and origin of black and red Magdalenian pigments from Grottes de la Garenne (Vallée moyenne de la Creuse-France) : a mineralogical and geochemical approach of the study of prehistorical paintings, Journal of Archaeological Science 38, p. 1165-1172.

Lahlil, S., Lebon, M., Beck, L., Rousselière, H., Vignaud, C., Reiche, I., Menu, M., Paillet, P. et Plassard, F., 2012 - The first in situ micro-Raman spectroscopic analysis of prehistoric cave art of Rouffignac St-Cernin, France, Journal of Raman Spectroscop, DOI : 10.1002/jrs.4115.

Nougier, L.-R. et Robert, R., 1957 - Rouffignac ou la Guerre des mammouths, Paris, La Table Ronde 311 p., 33 fig.

Ospitali, F., Smith, D.C. et Lorblanchet, M., 2006 Preliminary investigations by Raman microscopy of prehistoric pigments in the wall-painted cave at Roucadour, Quercy, France, Journal of Raman Spectroscopy 37, p. 1063-1071.

Plassard, F., 2005 - Les grottes ornées de Combarelles, Font-deGaume, Bernifal et Rouffignac. Contexte archéologique, thèmes et style des représentations, Thèse de l'Université Bordeaux 1, France.

Plassard, F., à paraître - Les bouquetins de la Grotte de Rouffignac : un exemple de cohérence stylistique, Paléo.

Plassard, J., 1999 - Rouffignac, le sanctuaire des mammouths, Paris, Le seuil, 96 p., 96 fig.

Solé V.A., Papillon E., Cotte M., Walter Ph. et Susini J., 2007 - A multiplatform code for the analysis of energy-dispersive X-ray fluorescence spectra, Spectrochim. Acta Part B 62, p. 63-68. 